$$
b=\frac{k_{1} m\left(\Lambda_{1}-0.6 A_{2}-20\right)}{2 A_{2}+605}
$$

In equation (26) the value of $H$ is expressed in terms of quantities which are either known or easily determined experimentally, and, therefore, $k_{2}$ may now be obtained by substitution of the value of $H$, so found, in equation (16). The following application to the case of tartaric acid will serve as an illustration of the method, while numerous additional acids are similarly treated in Mr. Chandler's article. For tartaric acid, $k_{1}=970 \times 10^{-6} ; 1$ if $m=\frac{\mathrm{I}}{\mathrm{IO} 24}, A_{1}=\mathrm{I} 4 \mathrm{I}$ and $A_{2}=\mathrm{IO} 8$. 'Therefore, $a=-2.167 \times 10^{-4}, c=-6.480 \times 10^{-8}, H=117.6 \times 10^{-5}$ and $k_{2}=34.3 \times \mathrm{IO}^{-6}$. The ratio $k_{1} / k_{2}=28.3$.

[Contribution from the Kent Chemical Laboratory, University of Chicago.]

\title{
THE IONIZATION CONSTANTS OF THE SECOND HYDROGEN ION OF DIBASIC ACIDS.
}

BY E. E. ChandLer.

Received March 3. Ig08.

It is generally believed that dibasic acids ionize in two stages, thus:

$$
\mathrm{H}_{2} \mathrm{X} \rightleftarrows \mathrm{H}+\mathrm{HX} \text { and } \mathrm{HX} \rightleftarrows \mathrm{H}+\mathrm{X} \text {. }
$$

From a study of the conductivities of dibasic acids, Ostwald ${ }^{2}$ concluded that, excepting strong acids like oxalic, the second stage of the ionization did not take place to an appreciable extent at concentrations greater than milli-normal, since the primary ionization constant, $k_{1}$, as calculated from the relation,

$$
H \cdot H X=k_{1} \cdot H_{2} X,
$$

where the formulae $H, H X$, etc., represent molecular or ionic concentrations, was really a constant for all concentrations greater than $1 / 1024$ normal. For smaller concentrations the apparent value of $k_{1}$ as calculated on the basis of equation ( $\mathrm{r}$ ) ustually increased appreciably. This increase is the result of the ionization of the second hydrogen ion.

If the secondary ionization constant, $i . e$, the ionization constant of the second hydrogen ion of a dibasic acid, is called $k_{z}$, then

$$
H \cdot H X=k_{z} \cdot H X \text {. }
$$

The magnitude of this constant has been determined previously for a considerable number of acids by four entirely different methods.

Trevor ${ }^{3}$ determined the rate at which dilute solutions of acid salts of dibasic acids invert cane sugar, and from the results calculated the concentrations of the hydrogen ions in the solutions used. It was assumed

${ }^{1}$ Walden, Z. physik. Chem., 8, 445 (1891).

"Ibid., 3, 281 (I 889 ).

3 Ibid., 10, 321 ( 1892$)$ 
that the dissociation of a salt like $\mathrm{NaHX}$ into $\mathrm{Na}$ and $\mathrm{HX}$ ions is practically complete in dilute solutions and that further ionization of the $\mathrm{HX}$ then yields $\mathrm{H}$ ions, the concentration of which governs the speed of in. version. A little later $\mathrm{A}$. A. Noyes ${ }^{1}$ developed a formula by means of which he calculated from Trevor's data the secondary ionization constants of the dibasic acids corresponding to the salts used by Trevor. Tower ${ }^{2}$ found values approximating those of Trevor and Noyes by the use of oxidation cells. Smith ${ }^{3}$ carefully repeated and extended Irovor's work, experiments being made to test the reliability of the method. Wegscheider ${ }^{4}$ obtained secondary ionization constants from tie conductivity of the free acids. A fourth, entirely distinct method was used by McCoy to find the secondary ionization constant of carbonic arid. "lhis method was later extended and applied to the study of sucinic acid." The method of McCoy as used for the latter acid, is based mithe following considerations. It was shown that when an acid sait 1. NaHS of a dibasic acid is dissolved in water it reacts thus:

$$
2 \mathrm{NaHX} \longleftrightarrow \mathrm{H}_{2} \mathrm{X}+\mathrm{Na}_{2} \mathrm{X} \text {. }
$$

The state of equilibrium in such a solution, as well as in one containing any atbitrary ratio of total acid and base, is governed by the relations expressed by equations ( 1 ) and (2), which by combination give

$$
\frac{H X^{3}}{H_{2} X \cdot X}=\frac{k_{1}}{k_{2}}
$$

In orcler to determine the state of equilibrium one must know the conentrations of the components. To find the concentration $\mathrm{H}_{2} \mathrm{X}$ the solulion may be shaken, until equilibrium is reached, with an immiscible ir partially miscible solvent, such as ether, in which the free acid is solu11. but the salt insoluble. The concentration of the acid in the ethereal lurer, multiplied by a factor. which is a constant for a given acid at a fixed temperature, gives the concentration of the free acid, in molecular form, in the aqueous solution. This factor is the partition coefficient of the free acid for water and ether, which in this case is the ratio of the concentration of the molecular $\mathrm{H}_{2} \mathrm{X}$ in an aqueons solution of the acid alone, to that of the total acid in an ethereal solution which is in equilibrium with the formet. If the total concentration of the base, $m$, is known for the aqueous solution containing $\mathrm{H}_{2} \mathrm{X}, \mathrm{NaHX}$ and $\mathrm{NaX}$, a determination of the total acidity, as shown by a titration, gives the remaining fuctor for the calculation of the concentrations of $\mathrm{HX}$ and $\mathrm{X}$. The for-

Z. physik. Chem, II, 495 (189.3).

2Ibid., 18, I7 (1895).

${ }^{3}$ Ibid., 25, I44, $193(1898)$.

4 Monatsh., 23, 599 (1902); 26, 1235 (1905).

s Am. Chem. J., 29, 437 (1903).

- Preceding paper. 
mulae by which these calculations are made are deduced in the preceding paper. It is there shown that in general

and

$$
H X=\frac{\alpha_{1} C}{2}+\sqrt{\left(\begin{array}{c}
\alpha_{1} C \\
2
\end{array}\right)^{2}-k_{1} \alpha_{1} H_{2} X}
$$

$$
X=\alpha_{2}\left(\frac{m+C}{2}-\frac{H X}{\alpha_{1}}\right) \text {. }
$$

$C$ is the equivalent acid concentration of the water solution minus $P$ times the equivalent acid concentration of the ether solution; $P$ is the partition coefficient; $\alpha_{1}$ and $\alpha_{2}$ are the degrees of ionization of the acid and neutral salts respectively, and $k_{1}$ is the primary ionization constant of the free dibasic acid; $m$ is the total concentration of the base (say sodium) in the solution. In most cases these formulas may be greatly simplified; where $k_{1} \alpha_{1} H_{2} X$ is very small compared with $\left(\frac{\alpha_{1} C}{2}\right)^{2}$,

In such cases

$$
H X=\alpha_{1} C \text { nearly. }
$$

$$
X=\alpha_{2}\left(\frac{m-C}{2}\right)
$$

The method just outlined was applied to the sodium salts of succinic acid by Professor McCoy, at whose suggestion I have studied the conditions of equilibrium in solutions of the salts of a number of other dibasic acids and from the results have calculated their secondary ionization constants. In the preceding paper it is shown how the secondary constants may also be calculated from the conductivities of the solutions of the acid and normal salts. I have also determined the conductivities of solutions of the salts of those acids studied by the partition method and from the results have obtained a set of independent values of the secondary ionization constants.

The experimental work described in the paper consists of three principal parts: the determination of

(I) The Partition Coefficients;

(2) The Equilibrium Constants;

(3) The Conductivities of the Salt Solutions.

\section{The Partition Coefficients.}

It is well known that a quantity of a substance shaken with a mixture of two immiscible or slightly miscible solvents at a fixed temperature is

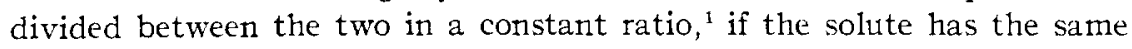
molecular composition in the two solvents. If there is in either solvent partial dissociation or association of the solute, then it is found that a

1 Berthelot and Jungfleisch, Ann. chim. phys. (4), 26, 390 (1874). 
constant ratio of concentrations obtains only for those portions of the solute having the same molecular composition in the two solvents. ${ }^{1}$

This ratio is known as the partition coefficient. I have found ether to be the best solvent in general for use in the determination of the state of equilibrium in solutions of dibasic acids and their salts. The experiments of McCoy showed that the salts of succinic acid are not taken up by the ether. In most cases ether dissolves the acids satisfactorily and gives for aqueous solutions of the free acid a constant partition coefficient when allowance is made for ionization, thereby indicating that in the ethereal solution the acid exists in the simple molecular form. In other solvents, $i$.e e, chloroform, many acids are partially associated. ${ }^{2}$ Moreover, an ethereal solution will readily separate from an aqueous solution, with which it has been shaken, while solutions of some of the other solvents tried do not readily do so. To purify the ether it was shaken with dilute sodium hydroxide solution, washed repeatedly with water, and finally distilled. The middle portion only was used. All of the partition coefficients were determined at $25^{\circ} \pm 0.0 \mathrm{r}$, except one series at $0^{\circ}$. The substances were shaken together in a plain cork-stoppered bottle in a thermostat, first by a mechanical shaker, until they had come to the temperature of the bath, and then violently for a few minutes by hand and, finally, allowed to settle for about half an hour. This procedure was found sufficient to insure the attainment of equilibrium and to effect a complete separation of the ethereal and aqueous layers. In order to remove a sample of the aqueous solution unmixed with ether, the top stem of the pipette was closed by the moistened forefinger of one hand as the pipette was passed through the ether layer; the body of the pipette was then grasped in the other hand. Sufficient heat was thus communicated to expand the enclosed air and expel the drop or two of ether that had entered the stem of the pipette. 'The solutions were titrated with standard barium hydroxide, phenolphthalein being used as indicator. Before the titration of the ethereal solution, water was added and the ether distilled off. Most of the acids used were products of the firm of C. A. F. Kahlbaum.

The results of the partition experiments with the free acids are given in the tables of Series I. The first column gives the molar concentration, $A$, of the aqueous solutions after treatment with ether. The degree of ionization, $\alpha$, of an acid was calculated from its primary ionization constant, $k_{1}$, by means of the equation

$$
\frac{\alpha^{2}}{(\mathrm{I}-\alpha) V}=k_{1} \text {. }
$$
(1897).

'Nernst, Z. physik Chem., 8, I 10 (1891). Hendrixson, Z. anorg. Chem., 13, 73

${ }^{2}$ Hendrixson, Loc. cit. 
For oxalic and dibromsuccinic acids and for dilute solutions of maleic acid $k_{1}$ is not constant; for oxalic and maleic acids, $\alpha$ was obtained by interpolation from the results of Ostwald $^{1}$ and for dibromsuccinic acid from those of Walden. ${ }^{2}$ The third column contains the values of the ratio, $p$, of the concentration of an aqueous solution of the acid to that of the corresponding ethereal solution. This ratio may be called the $\mathrm{m}$. corrected partition coefficient. The true partition coefficient, $P$, is the ratio of the concentration of the unionized or molecular acid in the two solvents. It is easily seen that $P=p(1-\alpha)$. The values containeri in the last coltumn are calculated from this relationship. N1l of the tables except one refer to partition between water and ether at $25^{\circ} \pm 0.01$. Table IV refers to succinic acid and these solvents at $0^{\circ}$.

\section{Series I.-Partition Coefficients.}

A.

0.3815

0.2767

0.2251

u. 1911

o. 1339

0.0887

0.0198

0.0103

0.0054

A.

0.1478

O.II2I

0.0862

0.0331

A.

0.1708

0.0582

0.0287

0.0217

0.0120

0.0059

0.0039

0.0023
TABLE I.-OXALIC AMII. ${ }_{10}^{B} k_{1}=38000$.

$\begin{array}{cc}100 \alpha . & 1 \\ 36 & 13.80 \\ 42 & 15.44 \\ 44 & 15.74 \\ 46 & 16.35 \\ 51 & 17.77 \\ 60 & 21.66 \\ 79 & +2.54 \\ 86 & 61.38 \\ 91.5 & 100.00\end{array}$

TABLE II. -Malonic Acid.

$$
{ }_{10} k_{1}=1580 \text {. }
$$

$100 \alpha$.

9.8

$p$

I I. 5

12. 6

19.6

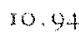

11.07

เ. . 28

12.22

TABLE III.-Succinic ACID.

$$
{ }_{10} k_{1}=66.5 \text {. }
$$
$100 \alpha$.

I. 9

$p$

3.2

7.7 .3

7.79

7.73

$4 \cdot 7$

$7.8 \mathrm{I}$

$5 \cdot 4$

7.2

10.0

12.2

15.6

7.95

8. 34

$8.4 \%$

8.7
I.

9.80

9. 79

68

0.82

9.83

7.59

7. 54

7.36

$-7.39$

7.37

3.55

7.39

$7 \cdot 4:$

'Z. physik. Chem. 3, 281 (1889).

'Ibid., 8, 479 (1897). 
SECOND HYDROGEN ION OF DIBASIC ACIDS.

TABLE IV.-SuccnNIC ACm at $0^{\circ}$

A.
0.0705
0.0702
0.0374
0.0200
0.0126
0.0116
0.0063
0.0039

A.

0.00998

0.00702

0.00480

0.00284

0.00179
$100 \alpha$.

3.0

3.0

4. I

5.6

7.0

$7 \cdot 3$

9.8

12.2 $p$.

$4 \cdot 43$

4.45

4.43

4.59

4.64

4.68

4.67

4.85
P.

4.30

$4 \cdot 31$

4.25

4.33

$4 \cdot 32$

$4 \cdot 33$

4.21

4.26

$4 \cdot 30$

TABLE V.-PIMELIC ACID.

$$
10^{\circ} k_{1}=32.3 \text {. }
$$

I00 $\alpha$.

$p$.

P.

$\begin{array}{rr}5.54 & 0.7095 \\ 6.56 & 0.7170 \\ 7.87 & 0.7195 \\ 11.30 & 0.7480 \\ 12.60 & 0.7075\end{array}$

0.670

0.670

0.663

0.663

0.653

0.664

TABLE VI.-Glutaric AcId.

$$
{ }^{\circ} 0^{\circ} k_{1}=47.4 \text {, }
$$

A.

0.0280

0.0085

0.0072

0.0063

0.0056
$100 \alpha$.

4.0

7.2

7.8

8.3

8.9 $p$.

3.72

3.84

3.91

$3 \cdot 92$

3.93
P.

3. 57

3.56

3.60

3.59

3.58

$3 \cdot 5^{8}$

TABle VII.-SUBERIC AcID.

A.

0.00986

0.00544

0.00175

0.00084

0.00049

$$
{ }_{10}^{\circ} k_{1}=29.9 \text {. }
$$
$100 \alpha$.

$5 \cdot 3$
7.2
12.2
17.2
21.9

$p$.

0.215

0.228

0.246

0.258

0.274
P.

0.204

0.2 I I

0.216

0.214

0.214 
$A$.
0.00310
0.00178
0.00123
0.00096
0.00077
0.00064
0.00058
0.00051
0.00046
0.0003 .3

A.

0.00062

0.00058

0.00047

0.00036

A.

$0.026 \mathrm{I}$

0.0197

0.0131

0.0119

0.0085

0.0057

0.0056

A.

0.000398

0.000272

0.000263

0.000250
A.

0.00229

0.00163

0.00148
TABLE VIII.-AZELAIC ACID. ${ }_{10}{ }^{\circ} k_{1}=25 \cdot 3$. I00 $\alpha$.

8.6

$p$.

$P$.

I I. 2

0.0679

0.0621

I $3 \cdot 4$

0.0702

0.0623

$\times 5.0$

0.0718

0.0622

I6. 6

0.0747

0.0635

0.0627

18.0

0.0753

$0.064 \mathrm{I}$

18.8

0.0782

0.0645

19.9

0.0800

0.0649

20.9

0.0810

0.0651

24,1

$0.082 ;$

0.0659

0.0637

' lable IX.-Sebacic AcID.

$$
{ }_{10}^{6} k_{1}=23.8 \text {. }
$$

$100 \alpha$.

$p$.

$P$.

17.8

0.0213

O.OI 75

18.3

0.0213

0.0174

20.1

0.0221

0.0176

22.6

0.0232

0.0179

0.0176

'TABLI: X.-ORThophthalic ACID.

$$
{ }_{10} k_{1}=\mathrm{I} 210 \text {. }
$$

I00 $\alpha$.

$p$.

$P$.

19.4

0.809

0.637

21.9

0.822

0.642

26. I

0.873

0.645

27.2

0.894

0.651

28.4

0.932

0.667

36.7

0.996

$0.63 \mathrm{I}$

36.9

1. 006

0.635

0.644

'Table XI.-Metaphthalic ACIO. ${ }_{10} 0^{\circ} k_{1}=287$.

$\begin{array}{ccc}\text { roo } \alpha . & p . & P . \\ 56.2 & 0.0821 & 0.0359 \\ 62.7 & 0.0943 & 0.0352 \\ 62.9 & 0.0944 & 0.0350 \\ 64.1 & 0.0949 & 0.0341 \\ & & 0.0350\end{array}$

I'ABLE XII,-CAMPHORIC ACID. ${ }_{10}{ }^{\circ} k_{1}=229$. $100 \alpha$.

$p$.

$P$.

0.0350

0.0353

I 1.2

0.0387

0.0398

0.0357

II. 7

o. .0403

0.0353 
TABLE XIII.--ITACONIC ACID.

A. ${ }_{10} k_{1}=151$.

0.0615

0.0306

0.0161

0.0103

0.0091

0.0039

A.

0.0993

0.0486

0.0337

0.0253

0.0196

0.0143

0.0100

0.0054

A.

$0.027 \mathrm{r}$

0.0114

0.0096

0.0092

$0.004 x$

0.004 I

A.

0.0879

0.0283

0.0253

$0.013 \mathrm{x}$

0.0056

A.

0.0327

0.0305

0.0 .302
I00 $\alpha$.

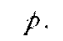
4. $b$
6.8
9.2
r. 4
13.8
21.5

2.99

3.06

3.15

3.20

3.22

3.46
TABLe XIV.-MaLeIC ACIS

$$
{ }^{\circ} 0_{1}=11700 \text {. }
$$

$100 \alpha$.

29.0

38.5

44.1

48.7

53.0

58.4

$63 \cdot 7$

73.1 $p$

g. (x)

11. 14

12. 20

$13.2 \%$

14.40

16.06

17.78

$2.3 \%$ $p$

2.83

$2.8 i$

2.88

2. 81

2.83

2.85

$\therefore .80$

$i$.

4. 82

0.88

0.82

1. 82

1. 78

6.69

0.45

6.38

6.78

'TABLE XV.-FUMARIC ACH.

$$
10^{\circ} k_{1}=930 \text {. }
$$

$100 \alpha$.

16.9
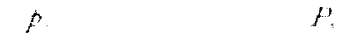

$-4.8$

a. 78.

0.650

26.6

1. 871

0.655

27.1

0.889

0.652

37.6

0. 89.3

0.648

37.6

1.053

0.658

1.040

0.650

a. 55 ?

TABLE XVI.-MoNoBromsUCCINIC ACID.

$$
100^{\circ} k_{1}=2780 \text {. }
$$

$$
\text { I00 } \alpha \text {. }
$$

$p$

$$
16.3
$$

0.413

0.344

26.8

0.479

o. 352

28.2

0.482

0.348

36.7

0.599

0.380

49.9

0.678

$\frac{0.340}{.345}$

'TABLE XVII.-DibromsuccinIC ACID.

$$
10^{\circ} k_{1}=34000 \text {. }
$$

$100 \alpha$. 
The results found for oxalic acid are remarkable; while the value of $p$ increases more than sevenfold within the range of concentrations used the value of $P$ is so nearly constant that the variations may reasonably be considered as due to experimental errors. Now the value of $\alpha$ used in calculating $P$ by means of the equation $P=p(\mathrm{I}-\alpha)$ is that determined from the conductivity of the acid. In its change' of ionization with change of concentration, oxalic acid does not follow exactly the Ostwald dilution law, in which respect it resembles salts and the stronger acids and bases. One of the most important problems connected with the ionic theory has been to decide whether the degree of ionization as determined in the ordinary way from the results of conductivity measurements of a good electrolyte is correct. Assuming the validity of the partition law, the constancy of the value of $P$ seems clearly to indicate the accuracy of the values of $\alpha$ used. The view that the correct value of $\alpha$ is given by conductivity measurements is in accord with the conclusion of A. A. Noyes, who has subjected all the other evidence on this poirt to a critical review. ${ }^{1}$ The constancy of the partition coefficient, $P$, is equally good for the balance of the acids, for most of which the dilution law holds true.

\section{Equilibrium in Solutions of Salts of Dibasic Acids; Determination of Equilibrium Constants.}

It has been shown that the state of equilibrium in a solution containing neutral salt, acid salt and free dibasic acid is represented by the equation

$$
\frac{H X^{2}}{H_{2} X \cdot X}=\frac{k_{1}}{k_{2}}
$$

The methods of determining the concentration of each of the constituents of such a solution was outlined in the introduction. In order to calculate the total concentration of the basic ion it is necessary to know the final volume of the aqueous solution. When water is shaken with ether, previously saturated with water, the volume of the aqueous layer in. creases; this increase in volume was in some cases measured directly in the manner indicated by McCoy for succinic solutions. In most experiments, however, it was found more convenient to determine the increase in volume in another way. It was found by experiment that when water or a dilute solution, such as was used in these experiments, was shaken with ether previously saturated with water, that the increase in volume of the aqueous solution always amounted, at $25^{\circ}$, to 7.8 per cent., and at $0^{\circ}$, to 15 per cent. The details of the experimental procedure for the determination of the equilibrium constant may be illustrated by an example taken from the work of glutaric acid. A quantity of pure glutaric acid sufficient to give a mixture of the acid and neu-

${ }^{1}$ International Congress, St. Louis, 1904, Vol. IV, p. 3 II. 
tral salts was added to $25.00 \mathrm{cc}$. of exactly normal sodium hydroxide and the solution diluted to $250 \mathrm{cc}$. Exactly $20 \mathrm{cc}$. of the resulting solution were shaken in a plain $250 \mathrm{cc}$. bottle with about $75 \mathrm{cc}$. of ether at a temperature of $25^{\circ} \pm 0.01$ until equilibrium was reached. This occurred within ten minutes. After the solution had stood in the thermostat for about thirty minutes longer and the ether and the aqueous layers had completely separated, a portion of each was removed, in the manner described for the determination of the partition coefficients, and titrated with standard barium hydroxide, the ether solution being first evaporated after the addition of water. In all cases phenolphthalein was used as indicator. $20 \mathrm{cc}$. of the aqueous solution required $10.70 \mathrm{cc}$. of 0.0972 normal barium hydroxide; $50 \mathrm{cc}$. of the ether solution required $18.60 \mathrm{cc}$. of 0.00992 normal barium hydroxide. 'l'herefore the (equivalent) acid concentration of the aqueous solution $=\frac{10.70 \times 0.0972}{20}=$ 0.0520 , and the (equivalent) acid concentration of the ethereal solution

$$
\begin{aligned}
& =\frac{18.60 \times 0.00992}{50}=0.00369 . \\
& C=0.0520-3.58 \times 0.00369=0.0388 . \\
& H_{2} X=0.5 \times 3.58 \times 0.00369=0.0066 .
\end{aligned}
$$

Since the aqueous volume is increased 7.8 per cent. by the absorption of ether, the total concentration of the sodium $=m=0.1 / 1.078=$ 0.0928 . $H X$ and $X$ may be calculated by the simplified formulas: $H X=$ $\alpha_{1} C$, and $X=\alpha_{2}\left(\frac{m-C}{2}\right) . \quad \alpha_{1}=0.79$ and $\alpha_{2}=0.70$. Therefore, $H X=0.0306$ and $X=0.0189$.

$$
\begin{gathered}
k_{1}=\frac{H X^{2}}{k_{2}}=7 \cdot 50 . \\
\text { IO }^{\circ} k_{1}=4 \cdot X \\
47 .
\end{gathered}
$$

Since the sodium salts of monobasic acids are, in general, very nearly equally ionized at equal concentrations, it seems probable that the tendency of an acid salt, $\mathrm{NaHX}$, to ionize into $\mathrm{Na}$ and $\mathrm{HX}$ must be about the same as that of the simpler salts, NaX. Consequently it has been assumed that $\alpha_{1}$, the degree of ionization of $\mathrm{NaHX}$, is equal to that of sodium acetate at the same concentration. The degree or ionization, $\alpha_{2}$, of a normal salt $\mathrm{Na}_{2} \mathrm{X}$ has been taken the same as that of normal sodium succinate at the same concentration. The equilibrium concentrations have been determined for solutions of the sodium salts of all the acids in Series I with the exception of oxalic, in which case the potassium salts were used on account of their greater solubility in water. Some difficulties were encountered in certain cases. The aqueous solution of potassium acid oxalate contains but a very small proportion of free acid and 
neutral salt. 'lhis condition is unfavorable for an accurate determina tion of the equilibrium concentrations and therefore of the ratio $k_{1} / k_{2}$. The same kind of difficulty in still greater degree appeared in the study of maleic acid solutions. It was necessary in each case to use a proportionately large quantity of the ether solution to obtain an accurate titration. Mono- and dibromsuccinic acids also present difficulties in that each is acted upon slowly by water, the first to give hydrobromic and malic acids, ${ }^{1}$ and the second to give hydrobromic and brommaleic acids. ${ }^{2}$ However, the action of water at $25^{\circ}$ is too slow to cause very appreciable errors.

\begin{tabular}{lcccccccc}
\multicolumn{7}{c}{ IABLE XVIII. GLUTARIC ACID } \\
$\alpha_{1}$. & $\alpha_{2}$. & $1 n$. & $C$. & $H_{2} X$. & $H X$. & $X$. & $k_{1} / k_{2}$. & $k_{1} / k_{2}$ \\
0.75 & 0.64 & 0.1855 & 0.0808 & 0.0167 & 0.0606 & 0.0335 & 6.6 & \\
0.75 & 0.64 & 0.1855 & 0.0807 & 0.0170 & 0.0605 & 0.0335 & 6.4 & 6.5 \\
0.78 & 0.695 & 0.1022 & 0.0405 & 0.0068 & 0.0316 & 0.0214 & 6.8 & \\
0.78 & 0.695 & 0.1022 & 0.0437 & 0.0089 & 0.0341 & 0.0203 & 6.4 & \\
0.78 & 0.695 & 0.1022 & 0.0448 & 0.0090 & 0.0349 & 0.0200 & 6.8 & 6 \\
0.79 & 0.70 & 0.0936 & 0.0464 & 0.0114 & 0.0366 & 0.0165 & 7.1 & \\
0.79 & 0.70 & 0.0936 & 0.0528 & 0.0165 & 0.0417 & 0.0143 & 7.4 & \\
0.79 & 0.70 & 0.0936 & 0.0529 & 0.0165 & 0.0418 & 0.0142 & 7.4 & \\
0.79 & 0.70 & 0.0936 & 0.0480 & 0.0119 & 0.0379 & 0.0160 & 7.6 & 7.4 \\
0.79 & 0.70 & 0.0928 & 0.0388 & 0.0066 & 0.0306 & 0.0189 & 7.5 & \\
0.79 & 0.70 & 0.0928 & 0.0464 & 0.0113 & 0.0366 & 0.0162 & 7.3 & 7.4 \\
0.81 & 0.73 & 0.0618 & 1.0084 & 0.0025 & 0.0068 & 0.0195 & 9.5 & \\
0.81 & 0.73 & 0.0618 & 0.0164 & 0.0151 & 0.0133 & 0.0166 & 7.1 & \\
0.81 & 0.73 & 0.0618 & 0.0269 & 0.0489 & 0.0218 & 0.0127 & 7.6 & \\
0.81 & 0.73 & 0.0618 & 0.0316 & 0.0763 & 0.0256 & 0.0110 & 7.6 & \\
0.81 & 0.73 & 0.0618 & 0.0344 & 0.0445 & 0.0279 & 0.0100 & 8.2 & \\
0.81 & 0.73 & 0.0618 & 0.0406 & 0.0150 & 0.0329 & 0.0077 & 9.4 & 8.1 \\
0.83 & 0.76 & 0.0464 & 0.0254 & 0.0064 & 0.0211 & 0.0080 & 8.7 & \\
0.83 & 0.76 & 0.0464 & 0.0261 & 0.0068 & 0.0217 & 0.0077 & 8.9 & \\
0.83 & 0.76 & 0.0464 & 0.0248 & 0.0070 & 0.0206 & 0.0082 & 7.4 & \\
0.83 & 0.76 & 0.0464 & 0.0248 & 0.0067 & 0.0206 & 0.0082 & 7.7 & \\
0.83 & 0.76 & 0.0464 & 0.0252 & 0.0068 & 0.0209 & 0.0081 & 8.0 & 8.2 \\
0.84 & 0.77 & 0.0366 & 0.0199 & 0.00526 & 0.0167 & 0.00643 & 8.3 & \\
0.84 & 0.77 & 0.0362 & 0.0197 & 0.00514 & 0.0165 & 0.00635 & 8.4 & \\
0.85 & 0.87 & 0.0316 & 0.0172 & 0.00490 & 0.0146 & 0.0562 & 7.8 & \\
0.85 & 0.87 & 0.0309 & 0.0172 & 0.00490 & 0.0146 & 0.00534 & 8.2 & 8.2 \\
0.90 & 0.83 & 0.0155 & 0.0083 & 0.00204 & 0.0075 & 0.00299 & 9.1 & \\
0.90 & 0.83 & 0.0155 & 0.0086 & 0.00236 & 0.0077 & 0.00286 & 8.9 & 9.0
\end{tabular}

Table XVIII gives the details of all experiments with glutaric acid. The symbols $m, C, H X$, and $X$ represent the respective concentrations in terms of gram equivalents per liter, while $\mathrm{H}_{2} X$ refers to gram molecules per liter. The simpler formulae, (6) and (7), have been used for calculating $H X$ and $X$ in all cases, except for oxalic and dibromsuccinic

1 'Yantur, J. Russ. Chem. Soc, 23, 339 (1892). Beilstein, Org. Chem., 1, $65^{8}$.

2Van't Hoff, Études de Dyn. Chem. Amsterdam, page 14. 
acids. Table XIX gives, in condensed form, the results for other acids; in each case several determinations were made at each concentration, as in the case of glutaric acid. The details are omitted in order to save space.

\section{TABLE XIX.}

Ratio of Ionization Constants, $k_{1} / k_{2}$, At Various Concentrations, $m$.

Oxalic......m $\quad 0.269$ r $0.13460 .07690 .0598 \quad 0.0448 \quad 0.0245$

$$
\begin{array}{lllllll}
k_{1} / k_{2} & 87 \mathrm{I} & 98 \mathrm{I} & 919 & 840 & 1087 & 880
\end{array}
$$

$\begin{array}{llllllllllll}\text { Malonic...... } m \quad 0.3134 & 0.1880 & 0.1180 & 0.0940 & 0.0784 & 0.0587 & 0.0470 & 0.0393\end{array}$

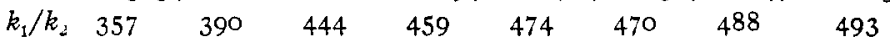

Succinic....m 0.18700 .08340 .03120 .01700 .0046

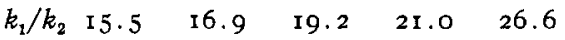

$\begin{array}{llllllllll}\text { Gilutaric.... } m \quad 0.1855 & 0.1022 & 0.0936 & 0.0618 & 0.0464 & 0.0362 & 0.0155\end{array}$

$\begin{array}{llllllll}k_{1} / k_{2} & 6.5 & 6.7 & 7.4 & 8.1 & 8.2 & 8.4 & 9.0\end{array}$

Suberic.....m 0.18550 .08240 .03110 .0169

$\begin{array}{lllll}k_{1} / k_{2} & 4.2 & 4.7 & 5.4 & 6.1\end{array}$

Pimelic... .. $m \quad 0.18590 .08330 .03090 .0169$

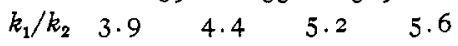

Azelaic.....m $m \quad 0.18550 .08240 .03110 .0169$

$\begin{array}{lllll}k_{1} / k_{2} & 42 & 4.8 & 5.2 & 5.8\end{array}$

$\begin{array}{llllllllllll}\text { Sebacic..... } m \quad 0.1855 & 0.0824 & 0.0337 & 0.0169 & 0.0046 & 0.0018 & 0.00092 & 0.00046\end{array}$

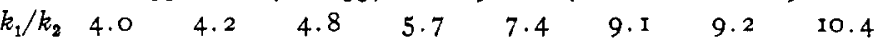

0 -Phthalic... m $\quad 0.1858 \quad 0.08250 .03090 .01700 .0089$

$\begin{array}{llllll}k_{1} / k_{2} & 165 & 200 & 240 & 265 & 290\end{array}$

$\begin{array}{lllll}m \text {-Phthalic... } m \quad 0.0926 & 0.0463 & 0.03 \text { I I }\end{array}$

$\begin{array}{llll}k_{1} / k_{2} & 5.3 & 6.6 & 6.5\end{array}$

Camphoric... $m \quad 0.09230 .04610 .01540 .0084$

$\begin{array}{lllll}k_{1} / k_{2} & 8.1 & 9.3 & \text { II.3 } & 13.2\end{array}$

Itaconic. . . m m $\quad 0.0926 \quad 0.0468 \quad 0.01560 .0084$

$\begin{array}{lllll}k_{1} / k_{2} & 33.7 & 38.9 & 46.1 & 57.0\end{array}$

Maleic......m $\quad 0.0926$

$k_{1} / k_{2} \quad 30000$

Fumaric....m $\quad 0.0926 \quad 0.0468 \quad 0.0156 \quad 0.0084$

Monobrom-

$\begin{array}{lllll}k_{1} / k_{2} & 18.3 & 20.3 & 25.8 & 30.0\end{array}$

succinic... $m \quad 0.0926 \quad 0.04630 .01560 .0084$

Dibromsuc-

$$
\begin{array}{lllll}
k_{1} / k_{2} & 46.0 & 54.0 & 69.0 & 91.0
\end{array}
$$

$\begin{array}{lllllll}\text { cinic...... } m \quad 0.0927 & 0.0463 & 0.0157\end{array}$

$$
\begin{array}{llll}
k_{1} / k_{2} & 10.9 & 13.5 & 12.6
\end{array}
$$

In discussing the results we may consider the influence of three factors upon the value of the equilibrium constant: (I) The effect of the ratio of the total base to the total acid in solutions of the salts where the concentration of the base is constant; (2) The effect of the total concentration of the base; (3) The effect of temperature. The experiments of McCoy on carbonates and succinates showed that, for constant concentra- 
tions of the total base, the value of $k_{1} / k_{2}$ is independent of the ratio of base to acid. My experiments lead to the same conclusion. If an aqueous solution of the pure acid salt (that is, one having equal molecular concentrations of base and acid) is shaken with ether the ratio of base to acid becomes greater than unity. In the experiments here described no attempt was made to maintain equal concentrations of base and acid in the aqueous solution; indeed in the case of glutaric acid the ratio was varied greatly in order to test the independence of $k_{1} / k_{2}$. The ratio of total base, $m$, to total acid in any experiment, is $\frac{\mathrm{C}+m}{2}+\mathrm{H}_{2} \mathrm{X}$.

the seventh experiment with glutaric acid this ratio is 1.044 ; in the tenth it is 1.282 ; and in the twelfth it is 1.644 . Such a high value of the ratio as the last is unfavorable to accuracy because of the exceedingly small titer of the ether solution; this doubtless accounts for the abnormal value found for $k_{1} / k_{2}$.

On the other hand, in the seventeenth experiment the ratio is 0.735 and it is interesting to note that in spite of the large excess of acid above that required to form the dry acid salt the solution contains an appreciable amount of the ion of the neutral salt. Here the great excess of acid is also unfavorable for an accurate determination of the constant; nevertheless, the value found is not far from the mean. In the first experiment with a sebacic acid the ratio of base to acid is 1.84 . Owing to the limited solubility of the acid in water, it is impossible to decrease this ratio very much at large concentrations of the base. Sebacic acid, however, is so much more soluble in ether than in water that there is always sufficient acid in the ether solution to make an accurate titration possible and no difficulty was found in getting concordant values of $k_{1} / k_{2}$.

I have found that $k_{1} / k_{2}$ increases with decreasing concentration of the total base. A similar result was found by McCoy for carbonates. Smith also observed a diminishing value of $k_{2}$ with dilution. This is equivalent to an increase of $k_{1} / k_{2}$ if $k_{1}$ is constant.

The values of $k_{1} / k_{2}$ have been found by inter-and extrapolation for the concentrations $0.1,0.01$ and 0.001 of total base with results shown in the following table. The last two columns contain the ionization constants of the first and second hydrogen ions. 'The values of $k_{2}$ were calculated from those of $k_{1} / k_{2}$ for 0.00 I 110 rmal concentration:

\begin{tabular}{|c|c|c|c|c|c|}
\hline & & $\begin{array}{c}\text { ABLE XX. } \\
k_{1} / k_{2} .\end{array}$ & & & \\
\hline & Conc. O.r. & Conc 0.01 & Conc, 0.001 & ${ }^{2} 0^{\dagger} k_{1}$. & ${ }_{10}^{6} k_{2}$ \\
\hline Oxalic............. & 930.0 & 930 & 930 & 38000 & 40.9 \\
\hline Malonic............. & 460 & 610 & 780 & I 580 & 2.03 \\
\hline Succinic. & 16.5 & $2.3 \cdot 5$ & 30.5 & 66.5 & 2. I8 \\
\hline Glutaric........... & 7.0 & 9.8 & 14.0 & $47 \cdot 4$ & $3 \cdot 3^{8}$ \\
\hline
\end{tabular}




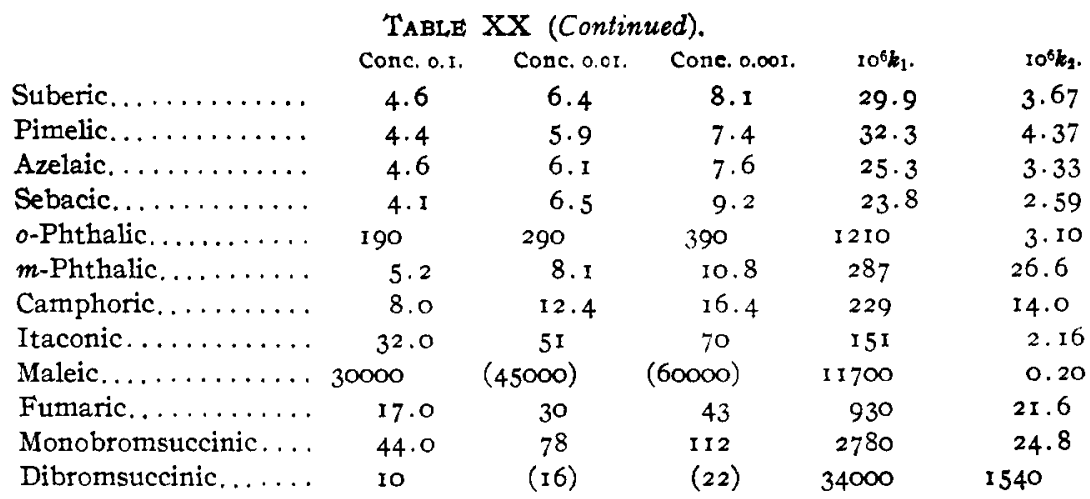

The values in parentheses, for maleic and dibromsuccinic acids, are uncertain.

The effect of temperature was determined by means of a series of experiments at $0^{\circ}$ with succinates. The following results were obtained:

TABLE XXI.

$\begin{array}{llllll}m \ldots \ldots \ldots \ldots \ldots \ldots & 0.1739 & 0.0869 & 0.0497 & 0.0290 & 0.0158 \\ k_{1} / k_{2} \ldots \ldots \ldots \ldots \ldots & 15.5 & 16.7 & 18.0 & 19.7 & 23.1\end{array}$

It will be seen that although the partition coefficient of succinic acid at $0^{\circ}$ (fourth table, Series I) is 4.30 , instead of 7.45 at $25^{\circ}$, yet the value of $k_{1} / k_{2}$ is practically the same at the two temperatures. This fact is in accord with many well-known results, which show that degrees of ionization and ionization constants are, for most substances, nearly the same at $0^{\circ}$ and at $25^{\circ}$.

It is interesting to note that $k_{1} / k_{2}$ for the dibromsuccinic acid is not greatly different from $k_{1} / k_{2}$ for succinic acid, although both $k_{1}$ and $k_{2}$ are enormously greater. Halogen substitution has therefore affected the dissociation of the two hydrogens about equally.

A series of experiments was carried out with sebacic acid, using chloroform instead of ether. The results of the partition experiments, conducted at $25^{\circ} \pm 0.0 \mathrm{r}$ are given in the following table. $A_{w}$ and $A_{c}$ represent the molecular concentrations of the acid in the water and chloroform solutions respectively. It is well known that there is association of the molecules of many substances when dissolved in chloroform. Allowance for $\alpha$ only, therefore, fails to make the values of $P$ constant. The smaller values of $P$ for the more concentrated solutions indicate, of course, a greater degree of association in the chloroform.

Tabie XXII.-Partition Coefficients of SEBacic Acid.

$\begin{array}{ccccc}A_{w .} & A_{c} & \alpha . & p . & P . \\ 0.00100 & 0.000963 & 14.3 & 1.04 & 0.89 \\ 0.00059 & 0.000438 & 18.2 & 1.36 & 1.09 \\ 0.00030 & 0.000176 & 24.5 & 1.70 & 1.29 \\ 0.00018 & 0.000087 & 30.3 & 2.08 & 1.45\end{array}$


The following table contains the results obtained in the determination of $k_{1} / k_{2}$ for sebacic acid by the chloroform method. The column headings have the same significance as in the tables where ether was used, but the column headed $A_{c}$ gives in addition the concentration of the acid in the chloroform. The experimental treatment is the same as that described for ether extraction except in two particulars. (I) The solubility of chloroform in water is so slight that the volume of the water solution is not changed when it is mixed with chloroform, so that the concentration of the total base is not altered thereby. (2) Inasmuch as there is no constant partition coefficient, its value for each experiment with a salt solution usually must be determined by interpolation. No partition experiment was made with the free sebacic acid in which the chloroform concentration was so great as that of the first experiment of the following table. The corresponding value of $P, 0.84$, was obtained by graphical extrapolation:

\begin{tabular}{cccccccccc}
\multicolumn{8}{c}{ TABLE XXIII.-SEBACIC ACID. } \\
$\alpha_{1}$. & $\alpha_{2}$. & $m$. & $C$. & $A_{C .}$ & $P$. & $H_{2} X$. & $H X$. & $X$. & $k_{1} / k_{2}$. \\
0.73 & 0.63 & 0.20080 & 0.02104 & 0.00148 & 0.84 & 0.00125 & 0.0154 & 0.0566 & 3.33 \\
0.79 & 0.70 & 0.09245 & 0.01182 & 0.00085 & 0.91 & 0.00077 & 0.0093 & 0.0282 & 4.01 \\
0.79 & 0.70 & 0.09245 & 0.01195 & 0.00094 & 0.89 & 0.00083 & 0.0094 & 0.0282 & 3.79 \\
0.85 & 0.78 & 0.03346 & 0.00454 & 0.00026 & 1.17 & 0.00031 & 0.0039 & 0.0113 & 4.34 \\
0.85 & 0.78 & 0.03346 & $0.0048+$ & 0.00033 & 1.14 & 0.00038 & 0.0041 & 0.0112 & 3.95 \\
0.88 & 0.82 & 0.01826 & 0.00269 & 0.00016 & 1.30 & 0.00021 & 0.0024 & 0.0064 & 4.29 \\
0.88 & 0.82 & 0.01826 & 0.00253 & 0.00014 & 1.33 & 0.00019 & 0.0022 & 0.0065 & 3.92
\end{tabular}

The first value of $k_{1} / k_{2}$ is uncertain on account of the uncertainty of the factor $P=0.84$. The remaining values of $k_{1} / k_{2}$ are almost constant. However, too much stress should not be laid on the constancy, as the method is involved by the phenomenon of association.

For an acid which is difficulty soluble in water, $k_{1} / k_{2}$ may be deter. mined in a manner still different from that described above. The solubility of the acid in pure water is first determined and allowance made for dissociation in the usual way. When the solid acid is present in excess the concentration of the undissociated portion of the dissolved acid in aqueous solution of the free acid is equal to its concentration when the salts also are present. It is well known that it is not easy to determine with accuracy the solubility of a difficultly soluble substance. About two months were spent in an endeavor to find the solubility of sebacic acid in water. Equilibrium between the dissolved and undissolved acid was sought by approaching $25^{\circ}$ from both a higher and a lower temperature. Rapid shaking was found to increase the solubility considerably. The result is probably to be explained by the comminution of the crystals by the greater agitation, it being apparently well established that smaller particles are more soluble than larger ones. ${ }^{1}$ Forty-four ex'Ostwald, Z. pilys. Chem., 34, 495 (1900); Hulett, Ibid., 37, 385 (1901). 
periments gave an average molar concentration of 0.00118 ; individual values ranged from 0.00125 to 0.00105 . At a molar concentration of 0.00118 , the degree of dissociation is 13.3 per cent. Therefore the concentration of the undissociated portion is $0.001 \mathrm{I} 8 \times 0.867=0.0010$. To filter the solution, it was drawn into a pipette by means of a filter pump through a closely packed cotton plug. It was then titrated. That error did not arise from imperfect filtration was shown by the fact that separately filtered portions of the same solution had the same concentrations.

To determine $k_{1} / k_{2}$ sodium hydroxide of known concentration was shaken with excess of acid, filtered as described, and titrated. The factors of equation (3) are determined in the same manner as for the partition method, except that the concentration, $\mathrm{H}_{2} \mathrm{X}$, remains 0.00 Io for all experiments. This method of determining $k_{2} / k_{2}$ for sebacic acid was not satisfactory. It was even more difficult to get a constant equilibrium between the acid salt, the neutral salt, the dissolved and undissolved acid, than between the last two alone.

The values found for $k_{1} / k_{2}$ averaged about 5 , but some values more than double this amount were found without my being able to assign any reason therefor.

The same method was also tried with suberic acid, but only one experiment was made. The molar concentration of its saturated solution was found to be 0.0144 , at which concentration the degree of dissociation is 4.4 per cent. and the molar concentration of the undissociated acid is o.or 38 . In a single experiment in which the total concentration of sodium was $0.20 \mathrm{I}$ the value 5.5 was found for $k_{1} / k_{2}$ for suberic acid. It is possible that an acid of about this solubility would give constant results and regret is expressed that more experiments were not made with suberic acid by this method.

\section{The Determination of the Secondary Ionization Constant of a Dibasic Acid by the Conductivity Method.}

The conductivity of the negative ion, $\mathrm{HX}$, of an acid salt, NaHX, cannot be found in the usual way on account of the ionic interactions of the solution of the acid salt. The values used by Ostwald ${ }^{1}$ and Bredig ${ }^{2}$ as the conductivities of $\mathrm{HX}$ ions, for the calculation of the degrees of ionization, from the conductivities of the free dibasic acids, were merely estimated from the composition of the ion.

I have found the approximate values of such ionic conductivities in the following manner: The concentrations of acid and neutral salts

1 Z. phys. Chem., 2, 840 (1888); 3, 281 (1889).

2 Ibid., I3, 191 (1894). 
and of free $\mathrm{H}$ ions were calculated for a dilute solution, say $\mathrm{N} / \mathrm{I} 000$, of the pure acid salt, by means of the value $k_{1} / k_{2}$ found by partition experiments. The observed equivalent conductivity of the solution of the acid salt diminished by the conductivity due to the neutral salt and $\mathrm{H}$ ions represents the conductivity of the ions of the acid salt. This difference, by division of a number representing the fraction of the sodium actually in the form of acid salt gave the true (hypothetical) equivalent conductivity of the acid salt. By subtracting from the latter value the known ionic conductivity of sodium the ionic conductivity of $\mathrm{HX}$ remained.

The results so obtained clearly revealed a very simple relationship. The conductivities of the $H X$ ions were all approximately proportional to those of the corresponding $X$ ions. The detailed results of these calculations are omitted, but the relationship discovered has been utilized to calculate the secondary ionization constants by a method entirely independent of the data of the partition experiments. The good agreement between the values of $k_{2}$ as found by the partition and conductivity methods serves, of course, as an equally satisfactory test of the accuracy of the law just announced.

'The same relationship between the conductivities of $\mathrm{HX}$ and $\mathrm{X}$ was observed when the conductivities of the $\mathrm{HX}$ ions were estimated in another way. The work of Ostwald ${ }^{1}$ and Bredig ${ }^{1}$ has served to show that the conductivity of an organic ion is dependent upon its composition. Univalent, isomeric ions are equally mobile, and univalent ions, composed of the same number of atoms, have practically the same conductivity. One may therefore safely assume that the conductivity of any ion, $\mathrm{HX}$, of a dibasic acid, $\mathrm{H}_{2} \mathrm{X}$, is equal to that of an ion of the most nearly related monobasic acid. Thus the conductivity of the acid succinic ion, $\mathrm{HCO}_{2}, \mathrm{CH}_{2} \cdot \mathrm{CH}_{2} \cdot \mathrm{CO}_{2}$, may be considered equal to that of the ion of butyric acid, $\mathrm{CH}_{3} \cdot \mathrm{CH}_{2} \cdot \mathrm{CH}_{2} \cdot \mathrm{CO}_{2}$.

In Table XXIV, $\frac{1}{2} X^{\lambda}$ is the equivalent conductivity of the $\mathrm{X}$ ion of the corresponding dibasic acid; $\lambda$ is the equivalent conductivity of the negative ion of that monobasic acid most closely resembling the dibasic acid of the same line. The ratio, $\lambda / \frac{1}{2} \lambda_{X}$, is nearly a constant, the mean value of which is 0.595 ; or practically 0.6 . We may therefore consider that

$$
\lambda_{H X}=0.6 \times \frac{1}{2} \lambda_{X}=0.3 \lambda_{X} .
$$

It is readily seen that the value of $\lambda_{H X}$, last column, Table XXIV, calculated by this equation, does not differ greatly, in any case, from that of $\lambda$. 
TABLE XXIV.

\begin{tabular}{|c|c|c|c|c|c|}
\hline & $\frac{1}{2} \lambda x$ & IV. & $\lambda$. & $\lambda / \frac{1}{2} \lambda X$ & $0.3^{\lambda} X=\lambda_{H X}$ \\
\hline Oxalic.... & 72.4 & Acetic... & 41.6 & 0.575 & $43 \cdot 4$ \\
\hline Malonic. . . . . . . . . . . & 64.1 & Propionic......... & 36.8 & 0.574 & $3^{8} \cdot 5$ \\
\hline Succinic............ & 56.0 & Butyric.......... & 33.0 & 0.589 & 33.6 \\
\hline Glutaric. . . . . . . . . . & 51.0 & Valerianic......... & 31.0 & 0.608 & 30.6 \\
\hline Suberic. . & $44 \cdot 3$ & Phthaluric. ....... & 26.5 & 0.598 & 26.5 \\
\hline Azelaic. ............ & $43 \cdot 3$ & Phthalanilic....... & 26.2 & 0.605 & 26.0 \\
\hline Sebacic..... & 40.1 & & & & $24 \cdot \mathrm{I}$ \\
\hline Camphoric......... & 40.8 & & & & $24 \cdot 5$ \\
\hline$o$-Phthalic............ & 49.8 & Toluic. & $32 \cdot 1$ & $0.65 \mathrm{I}$ & 29.9 \\
\hline$m$-Phthalic.......... & 50.0 & & 32.1 & 0.642 & 30.0 \\
\hline Itaconic . . . . . . . . & 55.7 & Angelic... & $3 \mathbf{r} \cdot 5$ & 0.566 & $33 \cdot 4$ \\
\hline Maleic............ & 60.0 & Crotonic.. & $34 \cdot 5$ & 0.575 & 36.0 \\
\hline Fumaric............ & 60.6 & $\ldots \ldots \ldots$ & $34 \cdot 5$ & 0.569 & 36.4 \\
\hline Monobromsuccinic.... & 56.4 & Butyric......... & 33.0 & $0.5^{85}$ & 33.8 \\
\hline Dibromsuccinic . . . . . . & 55.2 & $" \quad \ldots \ldots \ldots \ldots$ & 33.0 & 0.598 & $33 . I$ \\
\hline & & & dea & 595 & \\
\hline
\end{tabular}

By means of the relationship just discussed, McCoy has shown in the preceding paper, how the concentration of hydrogen ions in the solution of an acid salt of a dibasic acid may be calculated from conductivity data. For the acid sodium salt of $\mathrm{N} / \mathrm{IO}_{2} 4$ concentration,

$$
H=a+\sqrt{a^{2}+b}
$$

where

$$
a=m \frac{\left(\Lambda_{1}-A_{2}\right)-k_{1}\left(0.7 A_{2}+\mathrm{I} 4 \mathrm{I}\right)}{2 \Lambda_{2}+605}
$$

and

$$
b=\frac{k_{1} m\left(A_{1}-0.6 A_{2}-20\right)}{2 \Delta_{2}+605}
$$

and $A_{1}$ and $A_{2}$ represent the observed equivalent conductivities of the acid and normal salts respectively, at $25^{\circ}$ and $\mathrm{N} / \mathrm{IO} 24$ concentration. The value of $H$ calculated by means of the preceding equations may be used to calculate $k_{2}$ by substitution in Noyes's equation : 1

$$
k_{2}=\frac{\left(k_{1}+m+H\right) H^{2}}{k_{1}(m-H)} \text {. }
$$

I have measured the conductivities of those acid and normal salts studied by the partition method. The results are given in Tables XXV and XXVI. The conductivities of the acid salts of oxalic and malonic acids, as well as of many of the neutral salts, have been measured previously by Walden ${ }^{2}$ and Bredig." My results agree well in general with those of Walden and Bredig, in so far as comparison is possible.

' Z. phys. Chem., 11, 495 (1893).

Ibid., 8, 433 (1891).

'lbid., 13, I9I (1894). 
TABle XXV.-Condectivities at $25^{\circ}$ in Reciprocal Ohms of Acid Salts-NaHX

\begin{tabular}{|c|c|c|c|c|c|c|c|c|}
\hline Dilution. & 32. & 64. & 128. & 256. & 512. & 1024. & 2048. & 4096. \\
\hline Oxalic............. & 97 & 109 & 123 & 139 & 158 & I 82 & $21 x$ & $25^{\circ}$ \\
\hline Malonic. . & 80.5 & 84 & 87 & 90.6 & $97 \cdot 3$ & 104.8 & 113 & 126 \\
\hline Succinic. . . . . . . . & 76 & 80 & $8,3 \cdot 3$ & 86.5 & 89.5 & $9+$ & IOI & I I I \\
\hline dutaric ............ & $73 \cdot 4$ & 78.7 & $82 .+$ & 85 & 88 & 91.6 & 98 & 106 \\
\hline Suberic............ & 68.9 & 72.5 & $75 \cdot 7$ & 78.3 & $8 I .5$ & 85 & $9 \mathrm{I}$ & 99 \\
\hline Azelaic . . . . . . . . & 68.9 & 72.5 & $75 \cdot 7$ & $79 \cdot 3$ & 81.5 & 85 & 89.8 & 97 \\
\hline Sebacic...... & & & 7.5 & -8 & So & 82.9 & 88.3 & 95 \\
\hline$o$-Phthalic........... & 70.6 & 75 & 80 & 8.5 & 92 & 100 & II 2 & 125 \\
\hline$m$-Phthalic $\ldots \ldots \ldots \ldots$ & & & & $91 \cdot 3$ & 100.6 & I 15 & I 36 & 171 \\
\hline Itaconic . . . . . . . . & 74 & 77.8 & $8 \mathrm{I}+\mathrm{t}$ & 85.2 & 89 & $9+.8$ & 102.6 & 116 \\
\hline Maleic.............. & 79 & 84 & 88 & 91.5 & 95 & 99.5 & 104 & IIO \\
\hline Fumaric .......... & 80.5 & 86.2 & 93 & 104 & 118.7 & I 40 & 170 & 20.5 \\
\hline Monobromsuccinic & 78.6 & 86 & 96.5 & 109 & 127 & $15 \mathrm{I}$ & 180,6 & 217 \\
\hline Dibromsuccinic . . . . . . & 150 & 187 & 2.31 & 280 & 3.31 & $38 \mathrm{I}$ & 422 & $+5^{6}$ \\
\hline
\end{tabular}

\begin{tabular}{|c|c|c|c|c|c|c|c|c|}
\hline Dilution. & 32. & 64. & 128. & 256. & $5+2$. & 1024. & 2048. & 4096. \\
\hline Dxalic. & 99 & 105 & I 10 & 115 & 118 & 120 & I 2 I & 122 \\
\hline Malonic............. & 91.7 & $9^{8.2}$ & 102.4 & 105.5 & 109 & 112 & II.t & I 15.5 \\
\hline uccinic . . . . . . . . . & 87.3 & $92 \cdot 3$ & 96.7 & 99.7 & 102 & $10+$ & $105 \cdot 5$ & 106 \\
\hline Slutaric. & 82.9 & 88.2 & 92.5 & 95 & 97 & 99 & IOI & 102 \\
\hline uberic . . . . . . . . . . & 76 & $8 \mathrm{I} .4$ & 85.3 & 88.1 & 90.6 & 92.3 & 94.5 & 96 \\
\hline Azelaic. ............ & 75 & 80 & $8+$ & 87 & 89 & $9 \mathrm{I}$ & 92 & 9,3 \\
\hline Sebacic .............. & & & 81 & $s_{+}$ & $86 .+4$ & 88 & 90 & 9I \\
\hline o-Phthalic............ & 81.5 & 86.5 & 91 & $9+$ & 96 & 98 & 99 & 100 \\
\hline$m$-Plithalic. & & & & 92 & 95 & 98 & rol & 105 \\
\hline taconic. & 86 & 90.6 & 95 & $9^{8} \cdot+$ & 102 & 104 & 105 & 106 \\
\hline Maleic . . . . . . . . . & 90 & 95 & 99 & 102.5 & 105.5 & 108 & 109 & $109 \cdot 5$ \\
\hline "umaric............ & $89 \cdot 5$ & 94.6 & 98,5 & 102 & 105 & ios & 110 & 112 \\
\hline Monobromsuccinic. . & 86 & 91 & 95 & 99 & 102 & 105 & 108 & II 2 \\
\hline Dibromsuccinic. & $8+$ & 89 & 94 & $9^{8}$ & IOI & 104 & 107 & IIO \\
\hline
\end{tabular}

Table XXVII gives the results calculated from these conductivities by equations (9) and (12):

\begin{tabular}{|c|c|c|c|c|c|}
\hline & $\Lambda_{1}$. & $\Lambda_{2}$. & ${ }_{10}{ }^{\circ} k_{1}$ & ${ }^{10} 0^{6} \mathrm{H}$ & ${ }^{10} k_{2}$ \\
\hline Oxalic ........... & I 82 & 120 & 38000 & 193 & 49 \\
\hline Malonic........... & 104.8 & I I 2 & I 580 & 35 & $2 . \mathrm{I}$ \\
\hline Succinic...... & 94 & 104 & 66.5 & $x 2.9$ & 2.7 \\
\hline Glutaric. & 91.6 & 99 & $47 \cdot 4$ & I I . 4 & 2.9 \\
\hline Suberic $\ldots \ldots \ldots \ldots$ & 8.5 & $92 \cdot 3$ & 29.9 & $7 \cdot 4$ & 1.9 \\
\hline Azelaic .......... & 85 & 91 & $25 \cdot 3$ & $7 \cdot 7$ & 24 . \\
\hline Sebacic...... & $82 \cdot 9$ & 88 & 23.8 & 7.6 & 2.5 \\
\hline$o$-Phthalic......... & 100 & 98 & 1210 & $44 \cdot 4$ & $3 \cdot 9$ \\
\hline$m$-Phthalic ....... & 115 & 98 & 287 & 67.9 & 24 . \\
\hline Itaconic $\ldots . . . \ldots$ & 94.8 & 104 & I5 I & 18.7 & 2.8 \\
\hline Maleic.......... & $99 \cdot 5$ & 108 & I I 700 & I $5 \cdot \mathrm{I}$ & 0.26 \\
\hline Fumaric......... & 140 & 108 & 930 & II 3 & 32 \\
\hline Monobromsuccinic. . & I 51 & 105 & 2780 & I 5 I & 39 \\
\hline Dibromsuccinic.... & 381 & 104 & .34000 & 675 & 1600 \\
\hline
\end{tabular}


Table XXVIII contains all of the values which I have found for the ionization constants of the second hydrogen ion of the dibasic acids studied, together with those found by Smith, Wegscheider and Trevor.

TABLE XXVIII.-SeCONDARY IONIZATION CONSTANTS, $10^{8} k_{2}$.

Partition. Conductivity. Smith, Wegscheider. Trevor.

\begin{tabular}{|c|c|c|c|c|c|}
\hline Oxalic .............. & $4 \mathrm{I}$ & 49 & $\ldots$ & . & I6.0 \\
\hline Malonic ............. & 2.0 & 2.I & I.O & IO & $\cdots$ \\
\hline Succinic . . . . . . . . . & 2.2 & 2.7 & $2 \cdot 3$ & $\cdots$ & $\cdots$ \\
\hline Glutaric . . . . . . . . & $3 \cdot 4$ & 2.9 & 2.7 & . & $\cdots$ \\
\hline Suberic $\ldots \ldots \ldots \ldots$ & $3 \cdot 7$ & I. 9 & 2.5 & $3 \cdot 3$ & $\cdots$ \\
\hline Pimelic............. & $4 \cdot 4$ & . & . & $\cdots$ & $\cdots$ \\
\hline Azelaic. . . . . . . . . & $3 \cdot 3$ & 2.4 & 2.7 & $\cdots$ & $\cdots$ \\
\hline Sebacic ............ & 2.6 & 2.5 & 2.6 & $\cdots$ & . \\
\hline$o$-Phthalic............ & $3 \cdot I$ & $3 \cdot 9$ & 1. 7 & . & . \\
\hline$m$-Phthalic.......... & 27 & 24 & . & . & 10.0 \\
\hline Camphoric........... & I 4 & . & 0.7 & $\cdots$ & . \\
\hline Itaconic $\ldots \ldots \ldots \ldots$ & 2.2 & 2.8 & 2.3 & 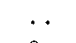 & , . \\
\hline Maleic............ & 0.20 & 0.26 & 0.4 & 8.0 & . \\
\hline 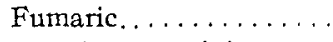 & 22 & 32 & I 8 & 29 & . \\
\hline Monobromsuccinic..... & 25 & 39 & . & 39 & . \\
\hline Dibromsuccinic ....... & 540 & $i 600$ & $\ldots$ & . & $\cdots$ \\
\hline
\end{tabular}

The methods of Trevor and Smith are similar, except that a different constant is used for the inversion effect of a completely dissociated acid. Their solutions were I/I 28 normal with respect to the acid salts and the experiments were carried out at $100^{\circ}$, as the speed of inversion was too slow at $25^{\circ}$. This should not materially effect the value of $k_{2}$, unless there was some decomposition of the acid at high temperature. Smith found that malonic acid suffered decided decomposition at $100^{\circ}$ and the value given is an interpolation. He also found that a neutral salt or even water caused quite as rapid an inversion as an acid salt, although in the former cases the rate was irregular. From these considerations, it is remarkable that values obtained by Smith's method agree so well with others.

The method of Wegscheider, using the free acid, is probably less accurate than the method using the acid salt. Even though the latter is complicated by the presence of the neutral salt. The former method is evidently more accurate for acids having a large value of $k_{2}$ than for those for which $k_{2}$ is small. The values found by Wegscheider for suberic, fumaric and monobromsuccinic acids agree very well with those $I$ have found. But his values of $k_{2}$ for maleic and malonic acids, which other experimenters find to be small, are certainly too large.

In conclusion, I wish to express my obligation to Dr. McCoy, under whose supervision the foregoing work has been carried out. 